\title{
El texto de Terencio: nuevos datos a la luz del Commentum Monacense*
}

\author{
Enara San Juan Manso \\ $\mathrm{UPV} / \mathrm{EHU}$ \\ enara.sanjuan@ehu.es

\section{The Text of Terence: New Information in Light of the Commentum Monacense}

\begin{abstract}
Dentro de la complejidad que caracteriza a la historia del texto de Terencio, los lemas del Commentum Monacense, conservado en el ms. München, Bayerische Staatsbibliothek, Clm 14420, ff. 79-144 (M), se nos presentan como un miembro más de la denominada familia $\theta$. Los lemas de M presentan, en concreto, a lo largo de las seis comedias de Terencio una extraordinaria similitud con el texto del ms. Paris, Bibliothèque nationale de France, lat. 7900A (Pc), lo cual obliga a postular la existencia de un modelo común inmediato para ambos mss. Las glosas de M y de Pc parecen indicar, por un lado, que ese modelo común inmediato estaría situado en una posición relativamente alta del stemma del texto de Terencio y, por otro, que, conteniendo aquel simultáneamente lecciones propias de las ramas $\delta$ y $\gamma$, habría sido cotejado con la ayuda de otro ms. con lecciones propias de cada una de las ramas.
\end{abstract}

Palabras clave: Terencio, texto, transmisión, Commentum Monacense.
Within the complex history of the text of Terence, the lemma of the Commentum Monacense, transmited in the MS. München, Bayerische Staatsbibliothek, Clm 14420, f. 79-144 (M), belong to the so-called $\theta$ family. The lemma of $\mathrm{M}$ are, throughout the six comedies of Terence, so similar to the text of the MS. Paris, Bibliothèque nationale de France, lat. $7900 \mathrm{~A}(\mathrm{Pc})$ that an immediate common model must be postulated. The glossa of M and Pc suggest, on the one hand, that this model would be located in a relatively high position of the stemma of the text of Terence and, on the other hand, that the model, simulteneously containing readings of the $\delta$ and $\gamma$ branches, would habe been collated with the help of another MS. with specific readings of each branch.

Key words: Terence, text, transmission, Commentum Monacense.

* GIU10-19 UPV/EHU; UFI11/14 UPV/EHU. Quisiera agradecer los consejos del profesor Íñigo Ruiz Arzalluz y de los evaluadores externos que han revisado el artículo para su publicación en esta revista. 


\section{Preliminar}

La historia del texto de Terencio pasa por ser una de las más complicadas de la filología latina ${ }^{1}$. En el origen de este hecho está, en cierta medida, la gran difusión que tuvieron las comedias terencianas desde la Antigüedad tardía, tal y como demuestran los mss. que han llegado hasta nosotros. Estos se dividen en dos grupos: por un lado, está A, el único ms. de la Antigüedad que conservamos; por otro, tenemos el conjunto de mss. medievales, en ningún caso anteriores a la época carolingia y considerados todos ellos descendientes de la denominada recensión caliopiana, cuya fecha no ha podido ser precisada con seguridad todavía ${ }^{2}$. Dentro del grupo de mss. medievales se diferencian, a su vez, dos ramas principales: $\delta$ y $\gamma$. La rama $\gamma$ habría emergido a comienzos del s. IX en el norte de Francia, mientras la mayoría de los descendientes de la rama $\delta$ se sitúa en el oeste de Alemania; en este sentido, los mss. que mejor atestiguan las lecciones y las características de cada una de estas dos familias medievales son principalmente $D, G, L$ y p para la rama $\delta$ y C, Y y P para la clase $\gamma$. En la base de la distinción entre estas dos familias hay, fundamentalmente, tres razones: por un lado, la presencia en los mss. de una serie de errores propios de cada una de las dos ramas; por otro, la presencia en muchos de los mss. de la rama $\gamma$ de ilustraciones que remiten a un antiguo ciclo de miniaturas ${ }^{3} ; \mathrm{y}$, en tercer lugar, el hecho de que el orden de las comedias sea, en principio, distinto en los mss. de cada una de las ramas (concretamente, Andr., Ad., Eun., Phorm., Haut., Hec. en los de la rama $\delta$ y Andr., Eun., Haut., Ad., Hec., Phorm. en los de la rama $\gamma)^{4}$.

${ }^{1}$ En relación a la complejidad de la tradición del texto terenciano basta remitir a la conocida afirmación de Pasquali 1952, p. 373: «In nessun altro autore si palesa così chiaramente come qui in Terenzio non già l'errore ma il limite dei metodi lachmanniani». La bibliografía oportuna para esta complicada cuestión se encuentra en los estudios de conjunto más recientes: Reeve 1986, Grant 1986, Ceccarelli 1992, Victor 1996 y Victor y Quesnel 1999.

2 Aquí y en adelante citamos los mss. según las siglas recogidas en el epígrafe «SigLAS», donde se señalan los datos esenciales acerca de cada uno de ellos. Un resumen de las diversas propuestas que ha habido para la datación de la denominada recensión caliopiana puede verse en Grant 1986, pp. 18-42, y Velaza 2007, pp. 34-35 y 85 ss.; v. al respecto también Escobar 2008, p. 333 ss.

${ }^{3}$ Grant 1986, pp. 18-59, 136 y 155-159; Grant 1975, pp. 141-143.

${ }^{4}$ Se ha sostenido que el orden de la rama $\delta$ está establecido según un criterio alfabético, considerando para Phorm. el título de Epidicazomenos o Formio. Por su parte, el de 
A pesar de la división bipartita establecida en el grupo de los mss. medievales, lo cierto es que, como consecuencia de continuos procesos de contaminación, la mayoría de los mss. medievales contiene simultáneamente lecciones particulares de ambas ramas ${ }^{5}$. Por ello, se habla a menudo de un grupo de mss. pertenecientes a una recensio mixta $(\mu)$, sobre cuyos miembros y relación con las ramas $\delta$ y $\gamma$ no hay acuerdo: Grant 1986, p. 137, por ejemplo, define los mss. pertenecientes a este grupo como mss. de la rama $\gamma$ que contienen un texto contaminado por mss. de la clase $\delta$ e incluye en el grupo mss. como E y v; otros autores, en cambio, ofrecen una definición más genérica de los mixti, sin llegar a definir los mss. que integrarían el grupo ${ }^{6}$. Los datos que impiden clasificar los mss. de manera clara en una de las dos ramas señaladas afectan también al orden de las comedias, ya que algunos de los mss. que se insertan en la rama $\delta$ siguen el orden propio de la clase $\gamma$ y no, en cambio, el de la rama $\delta$; Grant 1975, p. 152, supone que el ejemplar del que descienden los mss. de la rama $\delta$ debía de seguir el orden de la clase $\gamma$ y que, por tanto, el orden de las co-

la rama $\gamma$ muestra una sucesión cuyo origen se ha explicado hasta ahora de dos maneras: o bien como reflejo de un orden en el que las cuatro obras compuestas por Terencio según el modelo de Menandro preceden a las dos que toman como modelo a Apolodoro; o bien como consecuencia de una edición en dos volúmenes con las comedias dispuestas en origen como en A - es decir, Andr., Eun., Haut. en el primer volumen y Phorm., Hec., Ad. en el segundo-, en la que las comedias del segundo volumen pasaron a presentarse según una ordenación alfabética (Velaza 2007, pp. 35 y 67-68). Como recuerda Velaza 2007, p. 68 ss., Donato, Eugrafio o Prisciano atestiguan ordenaciones diferentes a las señaladas; v. al respecto también Sabbadini 1897, pp. 318-319; Grant 1973, pp. 88-103; Riou 1973, p. 88 n. 1; Villa 1984, pp. 2 y 200-201.

${ }^{5}$ Grant 1986, p. 102. Victor 2003, p. 353, afirma, en este sentido, que «the traditions that most defy stemmatic description are indeed those of poets: Lucan, Juvenal, Virgil and Terence ... Persius, Statius, Juvencus and Sedulius. They most often have been copied from several books at once»; v. al respecto la propuesta de Victor 2003, p. 347 ss., acerca de las prácticas seguidas por los copistas de textos clásicos en verso.

${ }^{6}$ Victor 2007, p. 2, por ejemplo, afirma que muchos de los mss. utilizados hasta fecha reciente en las ediciones del texto de Terencio - en concreto, F, E, G, L, v, $\varepsilon, \eta$, b y e- han sido objeto de contaminaciones. Velaza 2007, p. 131, por su parte, describe la situación en los siguientes términos: «La atribución de los códices datables entre los siglos IX y XII a las familias $\delta$ y $\gamma$ solo es sencilla en algunos casos, mientras que el fenómeno de la mixtificación supera con creces el esquema, propuesto por algunos especialistas, de una familia mixta $\mu$ de la que derivarían $E$ y $F$, para adquirir dimensiones de generalización entre los siglos XIII y XV». 
medias de la rama $\delta$ es un desarrollo secundario a partir del orden de la clase $\gamma^{7}$. Sin embargo, se ha defendido la mayor antigüedad del orden de la rama $\delta$, entre otros, a partir de la información proporcionada por Don., Vita 3: «... quamuis Volcatius $<$ in $>$ dinumeratione omnium ita scribat: "sumetur Hecyra sexta, exilis fabula" $\rangle^{8}$.

\section{LoS LEMAS DE M y LA FAMilia $\Theta$}

El Commentum Monacense - que debe su nombre a $\mathrm{M}$ - es un comentario a Terencio formado probablemente en la segunda mitad del s. IX y constituido por un accessus y una colección de glosas encabezadas siempre por un lema extraído del texto terenciano9. Por lo que al orden de las comedias se refiere, el Commentum Monacense se alinea con la rama $\gamma$ pero es interesante señalar que la indicación de la uita donatiana acerca de la posición de Hec., además de con el orden de la rama $\delta$, se corresponde también con la disposición de las comedias que reflejan seis dísticos memoriales conservados en $\mathrm{M}$ a continuación del citado comentario ${ }^{10}$ :

${ }^{7}$ Posteriormente Grant 1986, pp. 134-135, afirma que «since the $\gamma$ order was by far the commonest sequence in medieval MSS, it is not impossible ... that the order of plays in $\mathrm{NPbPcVb}$ has resulted from the influence of the predominant $\gamma$ order on these MSS or on antecedents of them. The problem, however, is more apparent than real, if we remember that the stemma worked out in this chapter applies only to Eunuchus. Some of these MSS have clearly a $\gamma$ text for the other plays. Their antecedents may have the $\gamma$ order, which was retained even though for Eunuchus and the other sections of the text an exemplar with the $\delta$ text and order was used».

${ }^{8}$ Velaza 2007, pp. 71-76; Escobar 2008, p. 340.

${ }^{9}$ Se puede consultar el códice completo en la siguiente página web de la Bayerische Staatsbibliothek: http://daten.digitale-sammlungen.de/0003/bsb00036895/images/ [consulta 6-V-2013]. En relación al Commentum son imprescindibles los estudios de Rand 1909a y 1909 b y Villa 1984, pp. 1-65. Algunas glosas de M fueron editadas por Schlee 1893 y recientemente, contamos con la edición — también parcial— de Schorsch 2011; la edición íntegra del ms. - que recientemente hemos preparado - se encuentra en proceso de publicación bajo el título El Commentum Monacense a Terencio, Vitoria-Gasteiz, Universidad del País Vasco, 2014.

${ }^{10}$ Los dísticos, conservados en el f. 144 del ms., han sido editados por Peiper 1877, p. 517; por Riou 1971, pp. 224-225; por Billanovich 1974, p. 39; y por Silagi y Bischoff 1979, p. 688, que remiten a Billanovich 1974. No hemos encontrado motivos para modificar la edición de Billanovich. 
Andria quid portet, iuuenes quae sponte secuntur, serui quo pacto pelliciant dominos;

Eunuchi uerum quod sit thema, nomen et unde assciuit, fraudes quae fuerint iuuenis;

Eautontimorumenos quem fert cruciatum ecessu gnati, gaudia quae reditu;

Adelphis patruus filio sumpsisse nepotem, alter quod Demea rus coluisse patre;

Phormio consilii quid det Phedriae parasitus, Antipho sobrinae captus amore suae;

Sexta loco proprium quod traxit nomen Echira est tibi sat notum: cetera deinde uide.

El orden en el que se mencionan las comedias de Terencio en estos versos se corresponde también con el de algunos mss. del entorno de Petrarca, por lo que cabe pensar que, con gran probabilidad, ejemplares relacionados con este grupo de mss. circularon cerca del lugar de origen de $\mathrm{M}^{11}$. Lamentablemente, las pruebas que avalan esta hipótesis son solo indirectas, ya que no ha sobrevivido ningún ms. anterior al s. XIV que, estrictamente, presente las comedias en el mismo orden que los versos de $\mathrm{M}^{12}$ : el caso más revelador es Vb, que conserva - en este orden- el texto de Andr., Eun., Haut., Ad. y prácticamente la totalidad del texto de Phorm. pero no así los últimos versos de esta última comedia ni tampoco, por tanto, el texto de Hec. ${ }^{13}$. También cabe pensar que esta comedia ocupaba la última posición en Ot, ya que, de-

${ }^{11}$ Entre los mss. del entorno de Petrarca en los que las comedias aparecen en el citado orden, Ruiz Arzalluz 2007-2008, pp. 809-810 n. 89, menciona Pm y Mp. A partir del desciframiento de un bifolio palimpsesto de M por parte de Bischoff 1972, se sitúa el origen de $\mathrm{M}$ en el monasterio de S. Faustino y Giovita de Brescia (norte de Italia). En base a diversos indicios, Villa 1979, p. 7, y Villa 1984, p. 25, denomina Brixiensis X y Vetus Brixiensis a los dos mss. anteriores que, en orden regresivo, se postulan para M; esta autora sospecha que las comedias aparecían en cada uno de ellos en el siguiente orden: Andr., Eun., Haut., Ad., Phorm., Hec. en el Vetus Brixiensis y Andr., Eun., Haut., Ad., Hec., Phorm. en el Brixiensis X.

${ }_{12}$ Además de los testimonios que señalamos a continuación, Prete 1982, p. 25, indica que Wog, con las comedias dispuestas según el orden de la clase $\gamma$, contiene la periocha de Phorm. en último lugar, lo cual «fa pensare che tale debe essere stato l'ordine del codice da cui il testo è stato preso»».

${ }_{13}$ Villa 1984, p. 35 n. 108, señala que el códice tiene una nota (s. XV) en el f. 120v que advierte: «Hic deficit quinta comedia que dicitur Hechyra», pero, puesto que la composición de los fascículos del ms. no apunta a ninguna pérdida de folios entre Ad. y Phorm., esta 
bido a la pérdida de los primeros folios, este ms. comienza con los versos 868-880 de Hec. Efectivamente, este hecho no prueba que el orden en el que aparecían las comedias en el ms. vaticano coincidiera con el de los versos memoriales de $\mathrm{M}$; no obstante, este ms. resulta interesante porque contiene al final del texto de Hec. un dístico («Ponti namque natans litus pertingere temptat | herbarum fessus carpere summa nitens») que parece anunciar metafóricamente el final de la labor del copista y que, precisamente, se encuentra al final de esa comedia también en Pc, cuyo contenido, como señalaremos a continuación, está estrechamente emparentado con $\mathrm{M}^{14}$.

Naturalmente, lo que nos atañe aquí no es averiguar cuál de los órdenes mencionados es más antiguo; quisiéramos, sin embargo, señalar que es más que posible que Pc estuviera relacionado con algún ejemplar en el que las comedias se presentaban en el mismo orden que en los versos memoriales de M y también, presumiblemente, que en $\mathrm{Vb}$. En este sentido, habría que señalar que precisamente $\mathrm{Pc}$ y $\mathrm{Vb}$ son los principales representantes de una misma familia de mss. denominada, dentro de la rama $\delta$, con la sigla $\theta^{15}$. Dentro de esta familia se han incluido estos tres mss.:

\section{Conv \\ $\mathrm{Fi}$ \\ $\mathrm{Ma}$;}

y cerca de Conv, Fi, Ma, Vb y Pc se han situado también los mss. que descienden del Terencio de Petrarca ${ }^{16}$.

autora da por segura la idea de que Hec. ocupara la última posición en ese ms., al igual que lo hace Grant 1986, p. 134.

14 Villa 1984, pp. 32-36. El dístico se conserva también en otros mss. que presentan las comedias según el orden de la rama $\gamma$.

15 Grant 1986, p. 126. El stemma propuesto por Grant difiere del propuesto por Marouzeau 1941-1949, I, p. 87, entre otras razones porque el estudio de Grant agrupa un corpus mayor de mss. - y geográficamente no tan limitado - que el utilizado por Marozeau; en este sentido, Victor 2007, pp. 1-12, es uno de los que ha lamentado que la base de mss. usada tradicionalmente por los editores modernos haya estado geográficamente limitada al norte de Francia y al sur de Alemania, sin tener en cuenta los mss. de Italia sobre todo. Grant 1986, p. 121 ss., señala, por otro lado, que la relación entre los mss. incluidos en el stemma de la rama $\delta$ varía a lo largo de las comedias.

${ }^{16} \mathrm{Al}$ menos por lo que respecta a las tres primeras comedias, ya que para las dos últimas los mss. que derivan del Terencio de Petrarca se han situado cerca de Pc, F y v; v. Ruiz Arzalluz 
Junto a todos ellos identificamos ahora en los lemas del denominado Commentum Monacense otro miembro de la denominada familia $\theta$. Si, como acabamos de señalar, el orden de los versos memoriales de $\mathrm{M}$ se relaciona, más o menos directamente, con $\mathrm{Pc}$ y $\mathrm{Vb}$, el texto que reflejan los lemas de M está también muy cerca del de Pc. En estudios anteriores, Grant 1978, p. 85, ha observado la gran similitud existente entre el texto de $A d$. de Pc y los lemas de M relativos a esa comedia y Grant 1978, pp. 88-89, también ha hecho notar la estrecha relación entre los tituli scaenarum de M y Pc, ya que ambos comparten incluso los errores de los títulos correspondientes a Andr. III 1 (459), Andr. III 3 (533), Eun. V 5 (840), Haut. I 2 (175) y Ad. III 4 (447), así como la presentación como titulus de un pasaje correspondiente a Phorm. 474 («GET. [CET. M] NESCIO. ANT.») ${ }^{17}$. A los datos aportados por

2007-2008, pp. 774, 778 y 789-792. En relación a esto último, debe destacarse que hay sobrados indicios de que el texto terenciano de Petrarca remonta a un modelo particularmente noble, lo que vendría a corroborar las conclusiones que mostraremos sobre la familia $\theta$. Tal circunstancia, por lo demás, no debe sorprender en absoluto, ya que son numerosos los casos en los que las tradiciones textuales rescatadas por Petrarca se han revelado como especialmente importantes; v. sobre esto Billanovich 1953, 1985, 1993 y 1994.

${ }^{17}$ Grant 1978 menciona, como excepción, que en M los títulos de las primeras escenas de $A n d r$. se omiten o se añaden en el margen. Cabe precisar al respecto que en $\mathrm{M}$ se omiten los tituli correspondientes a $A n d r$. I 1 (28) y $A n d r$. I 2 (172) y que se añaden en el margen los correspondientes a $A n d r$. I 5 (236) —repetido tras una glosa a $A n d r$. 267-, Andr. II 2 (338) y Andr. II 6 (432); los títulos relativos a las escenas $A n d r$. I 3 (206) y Andr. I 4 (228) no se pueden conocer debido a la pérdida de uno de los folios de M. En cuanto a los lemas del ms., anteriormente Schlee 1893, pp. 11-17, había señalado, a partir de un cotejo limitado a los tituli scaenarum de A, D, G, P, C, E y M, que el ms. más próximo a M era G, un ms. de la rama $\delta$, por lo que vinculaba el texto de $M$ con dicha familia. Sin aducir pruebas textuales, Rand 1909a, p. 386, supone que el Commentum Monacense surge junto a la rama mixta de la tradición de Terencio, lo cual explicaría las similitudes textuales entre M y los mss. de la rama $\delta$, así como el orden $\gamma$ de las comedias. Esta suposición es confirmada por Webb 1911, p. 65, quien, a partir de un cotejo de Haut. 522-904, llega a la conclusión de que los lemas de M pertenecen al grupo de mss. de la clase $\gamma$ contaminados con lecciones de la clase $\delta$ y propone la pertenencia de $\mathrm{M}$ al grupo $\mu$. Por lo que a las divisiones de escena se refiere, también Villa 1979, pp. 26-27, ha indicado que M coincide alternamente con las ramas $\delta$ y $\gamma$. Con la primera concuerda, en concreto, en la presencia de divisiones en $A n d r$. I 3 (206), Andr. V 6 (965) y Ad. III 5 (511), así como en la omisión de Eun. V 9 (1049) pero, en cambio, M mantiene ciertas divisiones de escena - las correspondientes a Haut. IV 7 (829), Haut. V 2 (954), Hec. V 2 (767) y Phorm. I 2 (51)- omitidas por mss. de la clase $\delta$ como $\mathrm{D}$, al tiempo que tampoco contiene la errónea división de escena introducida en Haut. V 2 (980) por mss. como D y G; asimismo, M coincide con la clase 
Grant, habría que añadir las omisiones de $A d$. I 1 (26), $A d$. II 3 (254), $A d$. III 3 (355), $A d$. V 4 (855), $A d$. V 7 (899) y $A d$. V 8 (924), la ortografía de $A n d r$. IV 4 (740) — «CHREMES SENES ...»- y $A d$. II 1 (155) — «SAMNIO ...»-, el error gramatical de Phorm. III 2 (485) — «... DORIA ...»-, así como el atributo de Sira en Hec. I 1 (58) — «... LENA ANVS»—. Asimismo, habría que señalar que, a pesar de estas coincidencias significativas, los tituli de M y Pc no siempre coinciden: Pc, por ejemplo, corrige el titulus de $A n d r$. III 2 (481) — «LESBIA-ANNVS ...»-, no contiene las omisiones de $A n d r$. IV 1 (625), Andr. V 2 (842), Ad. V 5 (882) —esta última propia de la clase $\gamma-$ y $A d$. V 9 (958), ni tampoco presenta errores gráficos en los tituli de Andr. V 1 (820) — «... SENES [SENEX M] DVO»—, Ad. II 4 (265) — «ADVLESCENTES [ADOLESCENTIS $M$ ] II ...»- y Ad. IV 3 (592) — «SENES II [SENEX $M$ ]»-; por el contrario, en Pc encontramos errores en los tituli de Eun. IV 7 (771) — «... CHREMETES ...»-, al tiempo que se omiten los correspondientes a $A d$. IV 6 (713), Ad. V 6 (889) y Phorm. IV 2 (591), el último de los cuales aparece en $M$ en el margen. En cuanto al texto, añadiríamos que la gran similitud observada por Grant entre los lemas de $\mathrm{M}$ correspondientes a $A d$. y el texto de Pc es extensible al resto de las comedias, puesto que ambos mss. coinciden casi plenamente en la alternancia de lecciones de la clase $\delta$ y $\gamma$ a lo largo de las seis comedias; por ejemplo, en casos como los siguientes ${ }^{18}$ :

$\gamma$ en la presencia de divisiones en Ad. IV 7 (719), Hec. V 2 (767) y Phorm. V 5 (829), así como en la omisión de $A d$. V 5 (882) y sigue también a mss. como P, C y F en la indicación —errónea - de una división de escena en Eun. V 4 (943). La autora destaca, en este sentido, que, a pesar de que en el ms. las comedias aparezcan según el orden de la rama $\gamma, \mathrm{M}$ no conserva las lagunas propias de los ejemplares más antiguos de la clase $\gamma$ (por un lado, la de Andr. 804-853 y, por otro, la de la periocha y el prólogo de Eun., este último con un orden especial en D, G y L).

${ }_{18}$ Puesto que el objetivo de este elenco es dar una indicación aproximativa al respecto, consideramos suficiente tomar como referencia la edición de Kauer, Lindsay y Skutsch 1988, designada en la tabla mediante la sigla KL. En dicha edición $\gamma$ denota el consenso entre $\lambda, C$, $\mathrm{P}, \mathrm{E}, \mathrm{F}, \mathrm{v}, \pi, \eta$ y $\varepsilon$, mientras $\delta$ lo hace en relación a D, G, L, p, V y $\alpha$. En efecto, algunos de estos mss. son incluidos por otros autores dentro del grupo $\mu$; recordemos que Grant 1986, p. 137, por ejemplo, incluye E y v en la denominada recensio mixta. No obstante, la lista que ofrecemos es válida para mostrar la estrecha relación entre las lecciones de $\mathrm{M}$ y de Pc dentro de las alternancias que existen en la tradición del texto de Terencio. En cuanto a las lecciones del aparato de la edición de Kauer, Lindsay y Skutsch 1988, remitimos a las observaciones de Ceccarelli 1992, p. 11. 
Andr. 677

Andr. 701

Andr. 742

Andr. 762

Andr. 774

Andr. 784

Andr. 915

Andr. 981

Eun. 51

Eun. 98

Eun. 503

Eun. 536

Eun. 598

Eun. 663

Eun. 688

Eun. 777

Eun. 894

Eun. 926

Eun. 985

Eun. 996

Eun. 1042

Eun. 1088

Haut. 80

Haut. 297

Haut. 319

Haut. 339

Haut. 406

Haut. 498

Haut. 691

Haut. 721

Haut. 764

Haut. 796

Haut. 800

Haut. 871

Haut. 930

Haut. 945

Haut. 964

Haut. 994

Haut. 1008

Ad. 168 adire periculum $\mathrm{M} \operatorname{Pc} \delta$

procliue $\mathrm{M} \mathrm{Pc}^{1} \delta \mathrm{E} \mathrm{v}$

apposuisti M Pc D G L

dico ego M Pc $\delta$

dabit magis $\mathrm{M}$ Pc D G L

necdum M Pc D L p

sit uir $\mathrm{M} \operatorname{Pc} \delta$

restat $\mathrm{M} P \mathrm{Pc} \delta \mathrm{E}$

perficies M Pc $\gamma$, schol. G, $\mathrm{L}^{2} \mathrm{p}^{2}$

hunc exclusti foras $\mathrm{M} \operatorname{Pc} \delta$

deducito M Pc D G L ${ }^{1} \mathrm{p}^{2}$

malam in rem $\mathrm{M} P \mathrm{Pc} \delta \mathrm{F} \mathrm{V}$

quis $\mathrm{M} \operatorname{Pc} \delta$

siet $\mathrm{M} P \mathrm{Pc} \delta \mathrm{E}$

uetus uietus $\mathrm{M} \operatorname{Pc} \delta$

istunc M Pc $\gamma\left(\right.$ istuc $\left.\varepsilon^{1}\right) \mathrm{p}$

dum is uenit $\mathrm{M}$ Pc Iov. $\delta \mathrm{E}$

omittam M Pc Iov. $\delta$ E v ${ }^{2}$

tunc M Pc $\delta$

inrumpere $\mathrm{M} \operatorname{Pc} \gamma \mathrm{D}^{2} \mathrm{p}$

tum tu M Pc $\gamma \mathrm{p}$

accede ubi uis M Pc $\delta \mathrm{E}$

est facto M Pc $\gamma$

scin tu hanc M Pc $\gamma$ (praeter $\left.\eta^{1}\right) \mathrm{L}$

mitte ista M Pc Iov. $\mathrm{D}^{1} \mathrm{G}^{1} \mathrm{p}$

istius modi $\mathrm{M}$ Pc D G p

expectate $\mathrm{M} P \mathrm{Pc} \gamma \mathrm{D}^{2} \mathrm{~L}$

hoc negotii M Pc $\gamma \mathrm{L} \alpha$

me M Pc $\gamma$ (praeter v), L

homo iste M Pc $\gamma \mathrm{L}$

mihi in mentem M Pc $\gamma \mathrm{D} \mathrm{L}$

malitia est M Pc $\gamma \mathrm{D}^{2} \mathrm{~L}$

hunc M Pc $\gamma \mathrm{D}^{2} \mathrm{~L}$

postulent $\mathrm{M} \operatorname{Pc} \gamma \mathrm{D}^{2} \mathrm{~L}$

pergam $\mathrm{M} P c \gamma \mathrm{D}^{2} \mathrm{~L}$

illius $\mathrm{M}$ Pc $\gamma \mathrm{D}^{2} \mathrm{~L}$

neque tu $\mathrm{M} \operatorname{Pc} \gamma \mathrm{D}^{2} \mathrm{~L}$

istam M Pc $\gamma\left(\right.$ praeter $\left.\eta^{1} \varepsilon\right)$

rogitem $\mathrm{M} \mathrm{Pc} \gamma \mathrm{L}$

tu iam $\mathrm{M}$; nunc tu iam $\mathrm{Pc} \delta$ periclum adire cett. apud KL

procliui cett. apud KL

posisti cett. apud KL; posuisti $\gamma \mathrm{p}$

ego dico cett. apud KL

mage dabit cett. apud KL

haec tu cett. apud KL; tu haec G

uir sit cett. apud KL

restet cett. apud KL

pertendes cett. apud KL

exclusti hunc foras cett. apud KL

adducito cett. apud KL

malam rem cett. apud KL

qui cett. apud KL

sit cett. apud KL

uietus uetus cett. apud KL

istum cett. apud KL

dum uenit cett. apud KL

mittam cett. apud KL

tum cett. apud KL

rumpere cett. apud KL

tu cett. apud KL

ubi uis accede cett. apud KL

factost $\mathrm{A} \delta$

scin hanc cett. apud KL

mitte cett. apud KL

huius modi cett. apud KL

om. cett. apud KL

negoti cett. apud KL

$m i$ cett. apud KL

iste homo cett. apud KL

in mentem cett. apud KL

summast cett. apud KL

eum cett. apud KL

postulet cett. apud KL

pergo cett. apud KL

eius cett. apud $\mathrm{KL}$

ut neque cett. apud KL

istanc cett. apud KL

rogem cett. apud KL

nunciam A; iam nunc tu $\gamma$ 
Ad. 181

Ad. 208

Ad. 228

Ad. 233

Ad. 256

Ad. 371

Ad. 492

Ad. 507

Ad. 588

Ad. 595

Ad. 626

Ad. 636

Ad. 791

Ad. 833

Ad. 878

Ad. 907

Ad. 950

Ad. 983

Hec. 23

Hec. 158

Hec. 184

Hec. 368

Hec. 373

Hec. 552

Hec. 584

Hec. 798

Phorm. 21

Phorm. 110

Phorm. 255

Phorm. 315

Phorm. 461

Phorm. 500

Phorm. 503

Phorm. 528

Phorm. 588

Phorm. 668

Phorm. 703

Phorm. 722

Phorm. 724

Phorm. 726 perges $\mathrm{M}$ Pc $\gamma \mathrm{D}^{2} \mathrm{~L}$

d. M; deputo Pc $\gamma \mathrm{G}$

illud $\mathrm{M}$ Pc A $\delta$

refrixit $\mathrm{M} \operatorname{Pc} \delta$ (praeter $\mathrm{D}^{2} \mathrm{G}$ )

certe M Pc $\gamma$ (praeter C)

est sane M Pc $\delta$ (praeter $\mathrm{p}$ )

uoster animus M Pc D G L $\varepsilon$

hoc M Pc $\gamma$, schol. D, G ${ }^{2} \mathrm{~L}$

otiose M Pc $\delta$, schol. v, $\varepsilon^{2}$

expostulent M Pc $\delta$

age $\mathrm{M} \mathrm{Pc} \gamma \mathrm{G}$

hęc acta M Pc D G L

scilicet $\mathrm{M}$ Pc $\gamma \mathrm{G} \mathrm{L}^{2}$

senectus affert M Pc $\gamma$

eo M Pc $\gamma \mathrm{D}^{2} \mathrm{~L}$

turbam M Pc $\gamma \mathrm{D}$

sit $\mathrm{M} \mathrm{Pc} \gamma \pi$

mi pater M Pc $\gamma$

ab arte musica M Pc $\gamma \mathrm{D}^{2} \mathrm{~L}$

se M Pc $\gamma \mathrm{L}$

a matre $\mathrm{M} P \mathrm{Pc} \gamma \mathrm{D}^{2} \mathrm{~L} \mathrm{p}^{2}$

d. $\mathrm{M}$; de repente $\mathrm{Pc} \gamma \mathrm{D}^{2} \mathrm{~L}$

ii $\mathrm{M}$ Pc $\gamma \mathrm{D}^{2} \mathrm{~L}$

nonne $\mathrm{M} \operatorname{Pc} \gamma \mathrm{D}^{2} \mathrm{~L}$

pietatis $\mathrm{M}$ Pc C P F v

referetque $\mathrm{M} P \mathrm{Pc} \gamma \mathrm{D}^{2} \mathrm{~L}$

ab ipso M Pc $\gamma \mathrm{D}^{2}$

scita inquit M Pc $\gamma$

responde hoc $\mathrm{M} P \mathrm{Pc} \gamma$

conspectum M Pc $\gamma$

exequar $\mathrm{M} \mathrm{Pc} \mathrm{A}^{2} \gamma \mathrm{D} \mathrm{L}^{2}$

duces M Pc $\gamma \mathrm{D}^{2} \mathrm{G}$

autem est $\mathrm{M}$ Pc $\gamma$

decepit $\mathrm{M}$ (detepit $\mathrm{M}) \mathrm{Pc} \gamma \mathrm{D}^{2}$

res mihi $\mathrm{M} \mathrm{Pc} \gamma$

perinde potius $\mathrm{M} \operatorname{Pc} \gamma \mathrm{D}^{2}$

sunt argentum $\mathrm{MPc} \gamma \mathrm{D}^{2}$

egressos M Pc $\gamma \mathrm{D}^{2}$

sat tuum $\mathrm{M}$ Pc C P E F ${ }^{1} \mathrm{v} \eta^{1}$

congruet $\mathrm{M} \operatorname{Pc} \gamma(i$ ex $e$ P) pergis cett. apud KL

puto cett. apud KL

illuc cett. apud KL

refrixerit cett. apud $\mathrm{KL}$

certo cett. apud KL

sane est cett. apud KL

animus uoster cett. apud KL

hic cett. apud KL

odiose cett. apud KL

expostulant $\gamma$

ac cett. apud KL; om. $\mathrm{D}^{1}$

acta haec cett. apud KL

ilicet cett. apud KL; licet D p

adfert senectus cett. apud KL

hoc cett. apud $\mathrm{KL}$; huc $\mathrm{D}^{1} \mathrm{G} \mathrm{p}$

turbas cett. apud KL

est cett. apud KL

pater mi cett. apud KL

$a b$... arte musica cett. apud KL

sese cett. apud KL

ad matrem cett. apud KL

repente cett. apud $\mathrm{KL}$

adueni cett. apud KL

nonne ea cett. apud KL

pietati cett. apud $\mathrm{KL}$

referet cett. apud KL

ab illo cett. apud KL

inquit scita $\delta$

hoc responde cett. apud $\mathrm{KL}$

aduentum $\mathrm{A} \delta$

sequar cett. apud $\mathrm{KL}$

ductes cett. apud KL; ductis A

est autem cett. apud KL

decipit cett. apud KL

mihi res cett. apud KL

perinde cett. apud KL; proinde A

sunt cett. apud KL

digressos cett. apud KL

satis est tuum cett. apud KL

conuenit A; congruit $\delta$ 
M y Pc muestran también lecciones que no aparecen atestiguadas en los aparatos críticos de las ediciones de Terencio, así como errores ortográficos o variantes comunes, entre otros ${ }^{19}$ :

Andr. $300 \quad$ ne de M Pc

Andr. 321 istud M Pc

Andr. 670 alia uia $\mathrm{M}$; alia uia adgrediemur $\mathrm{Pc}$

Eun. 39 falli se M Pc

Eun. $101 \quad$ haec $\mathrm{M}$; hec $\mathrm{Pc}$

Eun. 145 adducere M Pc

Eun. 163 donabat $\mathrm{M} \mathrm{Pc}$

Eun. 387 et illud M Pc

Eun. 480 hic M Pc

Eun. 583 apparat M Pc

Eun. 613 ista M Pc

Eun. 670 ut si M Pc

Eun. 692 rogo M Pc

Eun. 746 istius modi $\mathrm{M} \mathrm{Pc}$

Eun. 762 egenos $\mathrm{M} \mathrm{Pc}$

Eun. 763 interdum $\mathrm{M} \mathrm{Pc}$

Eun. 896 es obsecro M Pc

Eun. 922 sacrilegum perterrebo M Pc

Haut. 181 in Asia esse M Pc

Haut. 330 iam surdas M Pc

Haut. 331 hoc scies M Pc

Haut. 447 in uulgo M; uictum in uulgo $\mathrm{Pc}$

Haut. 707 prodis plane M Pc

Ad. 122 faciant M Pc

Ad. 132 esse M Pc

Ad. 141 haec omnia M Pc

Ad. 148 faciat M Pc

Ad. $230 \quad$ quaeque $\mathrm{M} \mathrm{Pc}$

Ad. 369 disrumptor $\mathrm{M}$; disruptor $\mathrm{Pc}$

Ad. $560 \quad$ ceptam M Pc

Ad. $561 \quad$ factum est $\mathrm{M} \mathrm{Pc}$ de edd.

istuc edd.

uia om. G; alia adgrediemur nia edd.

falli edd.

hic edd.

abducere edd.

dona dat edd.

illud edd.

haec edd.

apparatur edd.

istan $\mathrm{G}$; istac edd.

ut sibi edd.

roga edd.

eiusmodi $\gamma \mathrm{b} \mathrm{p}^{2}$; huiusmodi edd.

ego nos edd.

intus dum edd.

obsecro es edd.

perterrebo sacrilegum edd.

esse in Asia edd.

surdas iam edd.

scies edd.

uictum uolgo edd.

plane perdis A; plane prodis edd.

fiant edd.

est edd.

haec om. $\mathrm{F}^{1}$; omnia haec edd.

facit edd.

quae edd.

disrumpor (dirr-) edd.

emptam edd.

factum edd.

19 En la presente lista recurrimos a las ediciones de Kauer, Lindsay y Skutsch 1988, Umpfenbach 1870, Marouzeau 1941-1949 y Prete 1954 y señalamos mediante la abreviatura $e d d$. el consenso de los mss. utilizados en dichas ediciones que no mencionan ninguna variante distinta a las que se señalan; así también en adelante, salvo indicación expresa. 
Ad. $879 \quad$ eis M Pc

Phorm. 439 iam M; inpingi iam Pc

Phorm. 537 dixi M Pc

Phorm. 537 comite M Pc

Phorm. 601 pertimuit M Pc

Phorm. 821 parere M Pc

Phorm. 841 fortunate M Pc meis edd.

adpingam A; inpingan edd.

dixisti $\mathrm{D}$; dixti edd.

comiter edd.

pertimui edd.

parare edd.

fortuna edd.

M y Pc muestran, asimismo, errores significativos comunes con el grupo $\theta$; por ejemplo, la adición de uocat en Andr. 344, la adición de cum opus sit en Andr. 467, la omisión de nos en Eun. 456 (ecquid nos amas), la lectura credere ambos en Eun. 1069 y la trasposición en Haut. 184-19220. Estos errores se encuentran en b, el denominado Codex Bononiensis cuyas lecciones recoge Prete 1954 en su aparato ${ }^{21}$ :

Andr. 344 uocat? M; qui me uocat? o Pamphile Pc; qui me uolat o Pamphile b

Andr. 467 cum opus sit M;

qui me ..? o Pamphile edd. ne in mora illi sies cum opus sit $\mathrm{Pc} \mathrm{b}$

Eun. 456 quid amas $\mathrm{M}$; ec quid amas $\mathrm{Pc} \mathrm{b}$ ne in mora illi sis edd. (illi sies $\mathrm{D} \mathrm{C} \mathrm{C}^{1} \mathrm{~L}$ $\mathrm{p}$; sis illi $\mathrm{E}$ )

Eun. 1069 credere ambos $\mathrm{M} \mathrm{Pc} \mathrm{b}$ ecquid nos amas edd.

Haut. 192 entre Haut. 184 y Haut. 185 M Pc b ambos credere edd.

M y Pc también comparten con b lecciones que no aparecen atestiguadas en los aparatos críticos de las ediciones de Terencio, entre otras:

Andr. 261

Andr. 290 sollicitudo M Pc b

Eun. 298 eius $\mathrm{M} \mathrm{Pc} \mathrm{b}$

Eun. 562 fortunatum M Pc b

audias istuc $\mathrm{M} \mathrm{Pc} \mathrm{b}$ sollicitatio edd.

huius edd.

infortunatum edd.

audias edd.

${ }^{20}$ Ruiz Arzalluz 2007-2008, p. 775. La trasposición en Haut. 184-192 es señalada con anterioridad por Ceccarelli 1992, p. 18 n. 27.

${ }^{21}$ En relación al ms. cotejado por Prete 1954, v. asimismo Prete 1949-1950. La signatura que da Villa 1984, p. 304, es en realidad el número de inventario; actualmente el ms. ya no está, como dicen Prete 1949-1950 y Villa 1984, en la Biblioteca dell'Istituto di Filologia Classica, sino «en depósito» en la Biblioteca Universitaria di Bologna con la signatura que indicamos en el elenco final. 
Eun. 786 quid tibi uidetur M Pc; quid uidetur tibi $\mathrm{b}$

Eun. 871 hoc beneficium M; parerem hoc beneficium mihi Pc; hoc parerem mihi beneficium $\mathrm{b}$

Haut. 964 neque haec $\mathrm{MPc} \mathrm{b}$ quid uidetur edd.

parerem hoc mi beneficium edd.

neque ut haec edd.

Por tanto, M y Pc están estrechamente relacionados con la familia $\theta$, a la que, tal y como hemos señalado, pertenecería también el Terencio recuperado por Petrarca y de la que estaría cerca el Codex Bononiensis rescatado por Prete. Habría que subrayar, en cualquier caso, que $\mathrm{M}$ no presenta la trasposición de Andr. 243-246 advertida por Ceccarelli 1992 y compartida por toda la familia $\theta$-incluidos Pc y b_ ${ }^{22}$, lo cual podría indicar que el texto que reflejan los lemas de $\mathrm{M}$ está por encima de aquel que conservan el resto de los mss. de esta familia.

\section{LAS GLOSAS DE M}

A propósito de lo anterior, cabe prestar atención a las glosas de M, ya que estas contienen pistas acerca de la transmisión del texto que reflejan los lemas del ms. Al respecto Villa 1979, p. 28, señala — no sin cierta cautela- que $M$ «sembra conservare tracce di una revisione avvenuta, con l'aiuto di un esemplare $\gamma$, su di una base $\delta »$, lo cual resultaría aplicable al modelo común inmediato de $\mathrm{M}$ y $\mathrm{Pc}^{23}$ :

Andr. $362 \quad$ illoc: pro illuc M Pc illoc $\delta \mathrm{v} \mathrm{b} \quad$ illuc $\gamma$ (praeter v);

illo $\mathrm{G}$

Andr. 559 reducunt: pro reducant $\mathrm{M}$ Pc redducunt edd. redducant schol. C, E v b Andr. 771 quod: aliter cum M Pc quod $\delta$ quom edd.

Andr. 893-894 at tandem: aliter tamen M Pc at tandem D tamen edd.;

at tamen $\mathrm{G}$;

et tamen $\mathrm{V}$

Eun. 87 recta: pro recte $\mathrm{M} \mathrm{Pc}$ recta $\mathrm{D} \mathrm{G} \mathrm{E} \mathrm{C}^{2} \mathrm{~b}$ recte $\mathrm{C}^{1} \mathrm{P} \mathrm{p}^{2}$, schol. $\mathrm{v}$

${ }_{22}$ Ceccarelli 1992, p. 18 n. 27; Ruiz Arzalluz 2007-2008, p. 775.

${ }_{23}$ En la lista añadimos a la información aportada por Villa los datos de las ediciones de Kauer, Lindsay y Skutsch 1988 y de Prete 1954 e indicamos mediante edd. la lección adoptada en dichas ediciones. 
Haut. 746 harunc: pro harum M Pc

Haut. 947

Ad. 543

Phorm. 762 mite: pro dimitte $\mathrm{M}$;

dimitte Pc quid est: quid agis $\mathrm{M} \mathrm{P}$

sit: est $\mathrm{M}$ Pc harunc A edd. harum Iov., $\mathrm{P}^{2} \mathrm{~L}^{2} \mathrm{~F} \mathrm{p}^{2} \mathrm{v}$

mitte edd.

dimitte $\mathrm{L} F \mathrm{~F}$ b

quid est A D G p b quid agis (ais) C P E F v $\varepsilon$ sit edd. est C P E F b, schol. D

En cualquier caso, no todos los ejemplos que Villa aporta darían cuenta - como vemos - de una posible revisión llevada a cabo con la ayuda de un ejemplar $\gamma$ sobre una base $\delta$, puesto que ni todos los mss. que se encuentran en la tercera columna son mss. estrictamente pertenecientes a la denominada rama $\delta$, ni todos los mss. que encontramos en la cuarta pertenecen a la rama $\gamma$. En este sentido, se diría más bien que el modelo inmediato de $\mathrm{M}$ y Pc, conteniendo simultáneamente lecciones propias de las ramas $\delta$ y $\gamma$, podría haber sido cotejado con la ayuda de otro ms. con lecciones propias de ambas ramas. Esta posibilidad, además de adecuarse a las características de la mayoría de los mss. medievales, quedaría reforzada por la presencia en M y Pc de glosas como las siguientes ${ }^{24}$ :

Andr. 150 in obiurgandum: pro ad $\mathrm{M}$; in $^{\text {ad }}$ obiurgandum $\mathrm{Pc}$

Andr. 360 ipsus: pro ipse M Pc

Andr. 950 Pamphile: aliter Pamphili M Pc

Eun. 681 illum: ad illum M Pc

Ad. 242 conradet: aliter contrahet $\mathrm{M} \mathrm{Pc}$

Ad. 879 quoque: aliter quoquam M; quoque: quoquam Pc

Hec. 267 accusaturum: aliter accusaturum M; accusaturum: aliter accusat nirum Pc

Phorm. 189 uidissem: prouidissem M Pc

Phorm. 341 fit: uel sit M Pc
- $\quad$ ad obiurgandum edd.

ipsus $\mathrm{C}^{2}$ edd. $\quad$ ipse $\mathrm{C}^{1} \mathrm{P}$

Pamphile edd. Pamphili $\delta$

illum D G ad illum edd.

conradet edd. -

quoque edd.

accusat uirum edd.

uidissem edd. prouidissem Iov. $\delta$

fit edd. $\quad$ sit $\mathrm{C} \mathrm{P}$

Resulta también interesante observar que el modelo común inmediato de M y Pc podría haber tenido anotaciones interlineales de carácter textual. Estas no siempre habrían sido interpretadas de la misma manera por parte de los copistas y, según el modo en el que habrían sido entendidas, o bien se habrían copiado como glosa o bien, por el contrario, habrían pasado a formar

\footnotetext{
${ }^{24}$ Naturalmente, en estos casos no puede excluirse la posibilidad de que se trate de glosas personales antes que de glosas basadas en el cotejo de algún otro ms.
} 
parte del texto de manera independiente en uno y otro ms. Así, algunas de estas anotaciones se mantendrían como glosa o notas marginales en Pc y en M; por ejemplo:

Andr. 237 deum: atque hominum $\mathrm{M}$; pro deum fidem atque hominum $\mathrm{Pc}$

Andr. 576 consiliis: eorum M Pc

Andr. 885 uerbum uere: $s$. accidit in te M Pc

Andr. 951 mecum: s. Crito, s. ueni M Pc

Haut. 217 utetur patre: i. me M; utetur patre me $\mathrm{Pc}$

Haut. 256 uolebam: s. quos M Pc

Hec. 214 quę: me M Pc

Phorm. 739 meum nominat: s. nomen M;

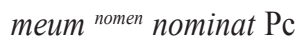

Phorm. 976 deęque: omnes M Pc pro deum fidem $\mathrm{C}^{1} \mathrm{P}$;

pro deum atque hominum fidem $\mathrm{C}^{2} \mathrm{D} \mathrm{B}$, schol. v;

pro deum fidem atque hominum $\mathrm{G} \mathrm{E} \mathrm{b}$

eorum consiliis edd.

uerbum uere in te accidit edd.;

uerum in te accidit $\mathrm{p}$;

uerbum in te uere accidit $\mathrm{G}$

mecum, Crito edd.;

mecum ueni Crito v

me utetur patre edd.

quos uolebam edd.

quae me edd.

meum nomen nominat edd.

deaque omnes edd.

En cambio - aunque este no es el único modo de interpretar los siguiente ejemplos - otras podrían haberse mantenido como nota interlineal o marginal solo en Pc; por ejemplo:

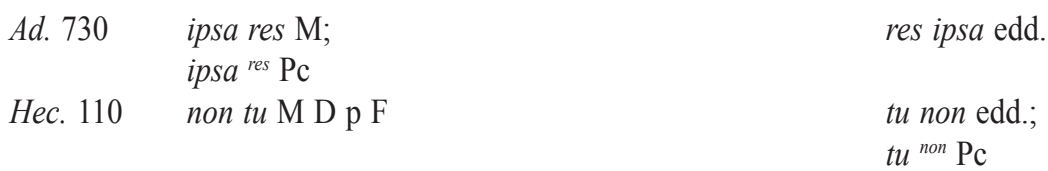

Hec. 851 boni quid sit $\mathrm{M} \mathrm{Pc}\left(\right.$ quid $\left.{ }^{\text {sit }} \mathrm{Pc}\right) \mathrm{F}^{1} \mathrm{v}^{1}(\mathrm{om}$. sit $\mathrm{F}$ v) edd. quid sit boni $\mathrm{C} \mathrm{P} \mathrm{L} \mathrm{E} \mathrm{B}$

Y, finalmente, otras podrían haberse mantenido como glosa solo en $\mathrm{M}$, bien debido a la inserción de las mismas en el texto de Pc, bien debido a la omisión de la glosa en Pc; de este modo — aunque no solo así- podrían interpretarse ejemplos como los siguientes:

Andr. 8 quam rem pro uitio s. dent $\mathrm{M}$ quam rem uitio dent edd.; uitio dent rem $\mathrm{D}$; quam rem pro uitio dent $\mathrm{Pc} \mathrm{E}^{2}$ 
Andr. 962 haec: cui $\mathrm{M}$

Haut. 92 meritum m.: s. est $\mathrm{M}$

Ad. 800 minus: s. mihi $\mathrm{M}$

Hec. 321 id: s. si $\mathrm{M}$

Phorm. 588 sollicitudini: s. est $\mathrm{M}$

Phorm. 714 testes: $s$. mihi $\mathrm{M}$

Phorm. 798 et locutus: quid M nunc haec Pc;

nunc cui haec cett. apud edd.;

cui nunc haec $\delta$ (L om. nunc) b

meritum est meum edd.;

meritum meum $\mathrm{Pc} b$

minus mihi Pc edd.

id si Pc;

id ${ }^{s i} \mathrm{D}^{2}$

sollicitudini est Pc edd.

mihi testis Pc edd.

et quid Pc; ecquid edd.

En relación con lo señalado, cabría también recoger aquí algunas variantes y glosas de M y Pc que, aunque pueden explicarse de diversas maneras ${ }^{25}$, al menos en algunos casos podrían entenderse en un sentido similar al que propone Villa 1979, pp. 29-32, en relación a M: a saber, como casos en los que glosas de $\mathrm{M}-\mathrm{y}$ ahora también de Pc — estarían en el origen de lecciones corruptas de mss. de los ss. X-XI, lo que situaría de nuevo el modelo común de M y Pc en una posición del stemma del texto de Terencio más alta de lo que, tácitamente, se ha supuesto hasta ahora:

Eun. 753 domo effer: et $\mathrm{M} \mathrm{Pc}$ domo ecfer edd. domo et effer (uel affer) $\mathrm{G}$ Eun. 777 portes: portas M Pc portes edd. portas D G LE

Eun. 854 ueritus: s. es M Pc ueritus A (es add. man. 2); ueritus es edd. ueritus $\mathrm{C}^{1} \mathrm{PF}^{1} \mathrm{v}^{1} \eta^{1} \varepsilon^{1} \mathrm{~b}$

Phorm. 164 certo: pro certo M Pc certo edd. certe Iov. G b; pro certo $\mathrm{E}$

Sin olvidar la gran coincidencia que existe entre las lecciones de $\mathrm{M}$ y Pc, cabría precisar que los lemas del primero no siempre concuerdan con el texto de Pc: en general, M presenta una lección singular frente a Pc, que - en tales casos - muestra habitualmente la lección vulgata, por ejemplo:

${ }^{25}$ Como afirma Grant 1986, p. 106, «many of the corruptions in the $\gamma$ and $\delta$ MSS of Terence do not betray themselves by their linguistic incomprehensibility. The corruptions have often occurred through interpolation, insignificant omissions, "regularization" of word order and syntax, and the removal or incorporation of archaisms». 


$\begin{array}{ll}\text { Andr. } 19 & \text { accusat } \mathrm{M} \\ \text { Andr. } 221 & \text { ciuem } \text { esse Atticam } \mathrm{M} \\ \text { Andr. } 247 & \text { fugere } \mathrm{M} \\ \text { Andr. } 257 & \text { illam } \mathrm{M} \\ \text { Andr. } 319 & \text { expectens } \mathrm{M} \\ \text { Andr. } 462 & \text { dicis } \mathrm{M} \\ \text { Andr. } 463 & \text { audio } \mathrm{M} \\ \text { Andr. } 521 & \text { id da } \mathrm{M} \\ \text { Andr. } 648 & \text { lectasses } \mathrm{M} \\ \text { Andr. } 761 & \text { territes } \mathrm{M} \\ \text { Andr. } 891 & \text { inliberi } \mathrm{M} \\ \text { Eun. } 94 & \text { penderet } \mathrm{M} \\ \text { Eun. } 184 & \text { moro } \mathrm{M} \\ \text { Eun. } 206 & \text { concedem } \mathrm{M} \\ \text { Eun. } 220 & \text { faciam opus } \mathrm{M} \\ \text { Eun. } 236 & \text { uideri } \mathrm{M} \\ \text { Eun. } 434 & \text { eris } \text { tu } \mathrm{M} \\ \text { Eun. } 522 & \text { mea } \mathrm{M} \\ \text { Eun. } 619 & \text { negares } \mathrm{M} \\ \text { Eun. } 728 & \text { uiuebar } \mathrm{M} \\ \text { Eun. } 787 & \text { cedes } \mathrm{M} \\ \text { Eun. } 791 & \text { accede } \mathrm{M} \\ \text { Eun. } 921 & \text { cognatione } \mathrm{M} \\ \text { Haut. } 79 & \text { ut sciam } \mathrm{M} \\ \text { Haut. } 132 & \text { decui } \mathrm{M} \\ \text { Haut. } 274 & \text { prospera } \mathrm{M} \\ \text { Haut. } 287 & \text { ex } \mathrm{M} \\ \text { Haut. } 298 & \text { dominicam } \mathrm{M} \\ \text { Haut. } 450 & \text { pernities } \mathrm{M} \\ \text { Haut. } 454 & \text { esne } \mathrm{M} \\ \text { Haut. } 544 & \text { querat } \mathrm{M} \\ \text { Haut. } 561 & \text { dicere } \mathrm{M} \\ \text { Haut. } 660 & \text { uiditne } \mathrm{M} \\ \text { Ad. } 43 & \text { fortunatam } \mathrm{M} \\ \text { Ad. } 233 & \text { uenit } \mathrm{M} \\ \text { Ad. } 262 & \text { putauit } \mathrm{M} \\ \text { Ad. } 314 & \text { illum } \mathrm{M} \\ \text { Ad. } 483 & \text { extorquere } \mathrm{M} \\ \text { Ad. } 592 & \text { laudarer } \mathrm{M} \\ \text { Ad. } 653 & \text { adeat } \mathrm{M} \\ & \end{array}$

accusant Pc edd.

ciuem Atticam esse Pc edd.

effugere Pc edd.

ullam Pc edd.

expetens Pc edd.

dicit Pc edd.

aut hic Pc edd.

id mihi da Pc edd.

lactasses Pc edd.

territas Pc edd.

liberi Pc edd.

penderem Pc edd.

moror Pc edd.

concedam Pc edd.

opus faciam Pc edd.

uideo Pc edd.

heus tu Pc edd.

ea Pc edd.

negare Pc edd.

uidebar Pc edd.

cederes Pc; caederes edd.

accedo Pc edd.

cognitione Pc edd.

ut faciam Pc edd.

decuit Pc edd.

propera Pc edd.

eius Pc edd.

dominam Pc edd.

pernitiem Pc; perniciem edd.

estne Pc edd.

queat $\mathrm{Pc}$ edd.

licere Pc edd.

uiuitne Pc edd.

fortunatum Pc edd.

uenis Pc edd.

putarit Pc edd.

illud Pc edd.

extorque Pc edd.

lauder Pc edd.

auehat Pc edd. 


\begin{tabular}{|c|c|c|}
\hline Ad. 711 & inpudens M & inprudens Pc edd. \\
\hline Ad. 778 & nominat hic $\mathrm{M}$ & hic nominat Pc edd. \\
\hline Ad. 865 & benedicter M & bene dicunt Pc edd. \\
\hline Ad. 899 & sanas $\mathrm{M}$ & sanctas $\mathrm{Pc}$ \\
\hline Ad. 951 & recedatur $\mathrm{M}$ & recte datur Pc edd. \\
\hline Hec. 71 & initium $\mathrm{M}$ & iniurium Pc edd. \\
\hline Hec. 87 & $u b i \mathrm{M}$ & $i b i$ Pc edd. \\
\hline Hec. 115 & amabant $\mathrm{M}$ & amabat Pc edd. \\
\hline Hec. 388 & si erit $\mathrm{M}$ & sient Pc edd. \\
\hline Hec. 398 & est $\mathrm{M}$ & esse Pc edd. \\
\hline Hec. 492 & distrahat $\mathrm{M}$ & distrahit Pc edd. \\
\hline Hec. 613 & subire $\mathrm{M}$ & abire Pc edd. \\
\hline Hec. 642 & nuptias $\mathrm{M}$ & nuntias Pc edd. \\
\hline Hec. 669 & hęc $\mathrm{M}$ & huic Pc edd. \\
\hline Hес. 792 & illam $\mathrm{M}$ & illas Pc edd. \\
\hline Hec. 824 & extimuis loco $\mathrm{M}$ & extimui ilico Pc edd. \\
\hline Phorm. 174 & intro $\mathrm{M}$ & de integro Pc edd. \\
\hline Phorm. 201 & ero $\mathrm{M}$ & eo Pc edd. \\
\hline Phorm. 228 & uallidiore $\mathrm{M}$ & callidiore Pc edd. \\
\hline Phorm. 382 & posses $\mathrm{M}$ & nosses Pc edd. \\
\hline Phorm. 425 & a domo $\mathrm{M}$ & domo Pc edd. \\
\hline Phorm. 630 & meum $\mathrm{M}$ & eum Pc edd. \\
\hline Phorm. 1031 & commemorarem $\mathrm{M}$ & commemorem Pc edd \\
\hline
\end{tabular}

Frente a casos como estos, se observan también en el texto de Pc errores ausentes en M; por ejemplo:

Andr. 612 ergo Pc

Eun. 863 furcifero (Eun. 862) cum se seruum presertim $\mathrm{Pc}$

Haut. 220 ubi om. Pc

Ad. 426 sic memento te sedulo Pc ego $\mathrm{M}$ edd.

praesertim cum se seruum $\mathrm{M}$

(presertim $\mathrm{M}$ ) edd.

ubi $\mathrm{M}$ edd.

sic memento sedulo $\mathrm{M}$ edd.

\section{Cuestiones finales}

Naturalmente, las divergencias entre el texto de M y Pc nos obligan a precisar la relación entre uno y otro. Grant 1975 , p. 89 , aboga por la posibilidad de que los dos desciendan de un modelo común muy próximo - con un intermediario entre este y $\mathrm{M}$, habida cuenta de los errores que presenta 
este último- pero admite no encontrar pruebas claras acerca de la relación entre ambos mss. y no descarta la posibilidad de que el Terencio del que procede $\mathrm{M}$ descienda de $\mathrm{Pc}$, hecho que explicaría los errores de $\mathrm{M}$ que no se encuentran en Pc. Villa 1984, p. 24 ss. (sobre todo 28-29), por su parte, defiende la idea de que ambos mss. reproducen la edición del texto de Terencio utilizada en el entorno de Milán y, ciertamente, nuestro examen no hace sino confirmar la estrecha relación señalada por Villa. Quedan, sin embargo, sin explicar las divergencias observadas entre Pc y M, en relación a las cuales habría que señalar que sobre el texto del primero parece haber sido llevada a cabo una revisión con el fin de corregir algunas de las lecciones del ms. que en origen habrían sido comunes a $\mathrm{M}$; en concreto, además de la corrección del titulus de Andr. III 2 (481) en Pc, señalaríamos los siguientes ejemplos ${ }^{26}$ :

\begin{tabular}{|c|c|c|c|}
\hline Eun. 731 & illarior $\mathrm{M}$ & ${ }^{h}$ ilarior Pc & hilarior edd. \\
\hline Eun. 1024 & iudicio $\mathrm{M}$ & indicio ex iudicio Pc & indicio edd. \\
\hline Eun. 1046 & gubernator $\mathrm{M}$ & gubernatrix ex gubernator Pc & gubernatrix edd. \\
\hline Haut. 645 & innocentior $\mathrm{M}$ & ignoscentior ex innocentior Pc & ignoscentior edd. \\
\hline Ad. 426 & latum $\mathrm{M}$ & la tum Pc & lautum edd. \\
\hline Ad. 485 & nequid $\mathrm{M}$ & $n e^{c}$ quid $\mathrm{Pc}$ & nec quid edd. \\
\hline Ad. 876 & sumpto $\mathrm{M}$ & sumptu ex sumpto Pc & sumptu edd. \\
\hline Hec. 458 & reliquid $\mathrm{M}$ & reliquid $^{t} \mathrm{Pc}$ & reliquit edd. \\
\hline Hec. 482 & aud $\mathrm{M}$ & ${ }^{h}$ aud $\mathrm{Pc}$ & haud edd. \\
\hline Hec. 751 & sancius $\mathrm{M}$ & 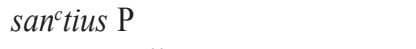 & sanctius edd. \\
\hline Hec. 792 & nullam $\mathrm{M} \mathrm{L}^{1} \mathrm{~F}^{1}$ & nam ex nullam Pc & nam edd. \\
\hline Phorm. 429 & a. $\mathrm{M}$ & ${ }^{h}$ abent $\mathrm{Pc}$ & habent edd. \\
\hline
\end{tabular}

Naturalmente, esta posible revisión no explicaría todos los casos en los que los lemas de M no coinciden con el texto de Pc, ya que no parece obligatoriamente aplicable ni a casos como estos:
Ad. 316
sublimen $\mathrm{M} \mathrm{G}$
Ad. 452
nihil $\mathrm{M}$ A D $\mathrm{D}^{1} \mathrm{G}$
Ad. 577
quidnam $\mathrm{M} \mathrm{D}^{1} \mathrm{v} \mathrm{b}$
Ad. 781
manus $\mathrm{M} \mathrm{D}^{2} \mathrm{E} F \boldsymbol{\eta} \varepsilon$
sublimem Pc edd. nihili Pc edd. quodnam Pc edd.
arg. Hec. 7
reuertitur $\mathrm{M} \mathrm{D}^{2} \mathrm{~L}$ E F $\eta \mathrm{b}$ manum Pc edd.
Phorm. 143
addit $\mathrm{M} \mathrm{G} \mathrm{F}^{2}$
reuenit $\mathrm{Pc} \mathrm{A} \mathrm{C} \mathrm{P}$
addet $\mathrm{Pc}^{2} \mathrm{~F}^{1} \mathrm{~V}$

\footnotetext{
${ }^{26}$ En cuanto a otros vestigios de posibles cotejos en Pc, v. Ceccarelli 1992, pp. 63-66.
} 
ni tampoco a casos en los que el copista de M podría haber introducido correcciones en los lemas ${ }^{27}$ :

$\begin{array}{lll}\text { Eun. } 990 & \text { exequi uel expedi } \mathrm{M} & \text { expedi } \text { Pc edd. } \\ \text { Haut. } 545 & \text { stot'idus } \mathrm{M} & \text { stolidus } \text { Pc edd. } \\ \text { Phorm. } 675 & \text { dan' } \text { ' } \mathrm{M} & \text { dant } \mathrm{Pc} \text { edd. }\end{array}$

Por tanto, se diría que las divergencias entre el texto de M y Pc se deben a procesos llevados a cabo, de manera independiente, sobre el texto que reflejan ambos mss.

Por otro lado, debido a la estrecha relación observada entre el texto de $\mathrm{M}$ y de Pc, podría plantearse la pregunta de si también las glosas que conservan estos mss. surgieron junto a la versión del texto de Terencio que ambos reflejan. El traer aquí esta cuestión no es banal, ya que el propio Grant 1978, pp. 84 y 90, ve la necesidad de aclarar si las glosas conservadas en $\mathrm{M}$ fueron escritas por primera vez para el Terencio del que depende M; en este sentido, a pesar de admitir la necesidad de estudios más amplios, Grant menciona los ejemplos de glosas relativas a $A d .401$ y a Phorm. 475 como indicio a favor de la idea de que las glosas de $\mathrm{M}$ hubieran sido compuestas en conjunción con la tradición del texto de Terencio de la que $\mathrm{M}$ deriva ${ }^{28}$. Además de los casos señalados por Grant, existen más ejemplos que apuntarían a la misma conclusión: la glosa relativa a Eun. 298 («O fortunatum] yronice»), por ejemplo, habría sido escrita para el texto «O fortunatum» (como el de M y Pc) y no, en cambio, para «O infortunatum» (como ocurre en otros mss. de Terencio); asimismo, la glosa relativa a Eun. 762 («Malo egenos] i. "magis uolo peregrinos", "sicut est Traso"》) habría sido escrita para el texto "Malo egenos» (como el de M y Pc) y no, en cambio, para «Malo ego nos» (como ocurre en otros mss. de Terencio). Sin embargo, no todas las glosas de M

${ }^{27}$ En relación con el ejemplo de Eun. 990, cabe señalar que el copista de M parece corregir sus propios errores también en ciertas glosas (por ejemplo, en las relativas a $A n d r$. 881, Eun. 604, Eun. 935 o Haut. 254), en cuyo caso se introduce la corrección mediante la abreviatura at (aliter).

${ }^{28}$ La glosa relativa a $A d .401$ («Abigam $\left.h(u n c) r(u s)\right]$ i. "compellam filium meum ire rus"») de M habría sido escrita para un texto en el que las palabras del lema eran pronunciadas por Démeas (como en Pc D G L E v ${ }^{1}$ ) y no por Siro (como en otros mss. de Terencio). Por su parte, la glosa acerca de Phorm. 475 («Nihil feci noui] i. "nihil nouitatis egi quod alii non fecissent"») habría sido escrita para un texto cuya lección era feci (como en M Pc E² F v) y no fecit (como en otros mss. de Terencio). 
ofrecen datos similares ${ }^{29}$, lo cual, además de ser comprensible habida cuenta de que los conjuntos de glosas no se transmiten en bloque de ms. a ms., pone en evidencia la relevancia que tienen también para el estudio de las glosas - y no solo para el de la tradición textual-nuestras conclusiones acerca de la filiación de los lemas de M.

\section{Conclusiones}

1. Dentro de la complejidad que caracteriza a la tradición del texto de Terencio, los lemas de M se nos presentan como un representante de la denominada familia $\theta$, a la que también pertenecen $\mathrm{Pc}, \mathrm{Vb}, \mathrm{Conv}, \mathrm{Fi}$, Ma o el ejemplar recuperado por Petrarca y de la que estaría cerca el Codex Bononiensis rescatado por Prete.

2. Las glosas de M y de Pc demuestran que el modelo común inmediato de ambos mss., conteniendo simultáneamente lecciones propias de las ramas $\delta$ y $\gamma$, habría sido cotejado con la ayuda de otro ms. con lecciones propias de ambas ramas. M y Pc contienen también glosas que, aunque podrían explicarse de diversas maneras, en algunos casos pueden entenderse como escolios que estarían en el origen de lecciones corruptas de diversos mss. de los ss. X-XI, lo que situaría el modelo común de M y Pc en una posición del stemma del texto de Terencio más alta de lo que, tácitamente, se ha supuesto hasta ahora. 3. A pesar de la extraordinaria afinidad en cuanto al texto y las glosas de M y Pc, el contenido de estos mss. no siempre concuerda entre sí, lo cual parece deberse en algunos casos al hecho de que, de manera independiente, se hubieran llevado a cabo procesos sobre el texto transmitido por dichos mss. Asimismo, M no presenta la trasposición de Andr. 243-246 advertida por Ceccarelli 1992 y compartida por toda la familia $\theta$, lo cual podría indicar que el texto que reflejan los lemas de $\mathrm{M}$ está por encima de aquel que conservan el resto de los mss. de esta familia.

4. La filiación de los lemas de $\mathrm{M}$ contribuye al conocimiento de la historia del texto de Terencio y ayuda a aclarar el sentido de determinadas glosas transmitidas en M y Pc junto a lecciones del texto diferentes en cada uno de los mss.

${ }^{29}$ Por ejemplo, la relativa a Haut. 684 («O mi Sire] congaudendo “miserum” eum uocat»), presumiblemente referida en origen al texto o misere (como el de Pc) y no al texto o mi Syre (como el de $\mathrm{M}$ y otros mss.). 


\section{BIBLIOGRAFÍA}

Billanovich, G. 1953: I primi umanisti e le tradizioni dei classici latini, Fribourg (ahora en Billanovich, G. 1996: Petrarca e il primo Umanesimo, Padua, pp. 117-141).

Billanovich, G. 1974: «Terenzio, Ildemaro, Petrarca», Italia Medioevale e Umanistica 17, pp. 1-60.

Billanovich, G. 1985: «Le tradizioni dei classici latini e la letteratura italiana tra Medioevo e Umanesimo», en Malato, E. (ed.), La critica del testo. Problemi di metodo ed esperienze di lavoro. Atti del convegno di Lecce 22-26 ottobre 1984, Roma, pp. 289-304.

Billanovich, G. 1993: «Ancora dalla antica Ravenna alle biblioteche umanistiche», Italia Medioevale e Umanistica 36, pp. 107-174.

Billanovich, G. 1994: M. Cortesi (ed.), Itinera. Vicende di libri e di testi, Roma, I-II.

Bischoff, B. 1972: «Das Güterverzeichnis des Klosters Ss. Faustino e Giovita in Brescia aus dem Jahre 964», Italia Medioevale e Umanistica 15, pp. 53-61.

Ceccarelli, L. 1992: Primi sondaggi sulla tradizione manoscritta di Terenzio, Roma. Escobar, Á. 2008: reseña de Velaza 2007, Exemplaria Classica 12, pp. 331-343.

Grant, J. N. 1973: «Г and the Miniatures of Terence», The Classical Quarterly 23, pp. 88-103.

Grant, J. N. 1975: «Contamination in the Mixed MSS of Terence: a Partial Solution?», Transactions of the American Philological Association 105, pp. 123-153.

Grant, J. N. 1978: «The Commentum Monacense and the Ms Tradition of Terence», Manuscripta 22, pp. 83-90.

Grant, J. N. 1986: Studies in the Textual Tradition of Terence, Toronto.

Kauer, R., Lindsay, W. M. y Skutsch, O. 1988: P. Terenti Afri Comoediae, Oxford.

Marouzeau, J. 1941-1949: Térence. Comedies, ed. J. Marouzeau, París, I-III.

Munk Olsen, B. 1982-2009: L'étude des auteurs classiques latins aux XI et XII siècles, París, I-IV/1.

Pasquali, G. 1952²: Storia della tradizione e critica del testo, Florencia.

Peiper, R. 1877: «Vermischte Bemerkungen und Mittheilungen zu römischen Dichtern zum Theil aus Handschriften», Rheinisches Museum 32, pp. 516-537.

Prete, S. 1949-1950: «Un nuovo codice di Terenzio», Rendiconti della Sessione della Accademia delle Scienze dell'Istituto di Bologna, Cl. scienze morali 5, pp. 208-229.

Prete, S. 1954: P. Terenti Afri Comoediae, Heidelberg.

Prete, S. 1982: «I manoscritti di Terenzio nella Biblioteca di Wolfenbüttel», en Prete, S. y Badalì, R., I Codici di Terenzio e quelli di Lucano nella Herzog August Bibliothek di Wolfenbüttel, Wolfenbüttel, pp. 9-36.

Rand, E. K. 1909a: «Early Mediaeval Commentaries on Terence», Classical Philology 4, pp. 359-389. 
Rand, E. K. 1909b: «Early Mediaeval Commentaries on Terence. Addendum», Transactions of the American Philological Association 40, pp. LXXII-LXXIII. Reeve, M. D. 1986²: «Terence», en Reynolds, L. D. (ed.), Texts and Transmission. A Survey of Latin Classics, Oxford, pp. 412-420.

Riou, Y. F. 1971: «Notes sur quelques manuscrits de textes classiques latins conservés à la Bibliothèque Vaticane», Revue d'Histoire des Textes 1, pp. 119-225.

Riou, Y. F. 1973: «Essai sur la tradition manuscrite du Commentum Brunsianum des Comédies de Térence», Revue d'Histoire des Textes 3, pp. 79-113.

Riou, Y. F. 1978: «Gloses et commentaires des comédies de Térence dans les manuscrits de la bibliothèque du monastère San Lorenzo el Real de l'Escorial», en Cambier, G., Deroux, C. y Preaux, J. (eds.), Lettres latines du Moyen Âge et de la Renaissance, Bruselas, pp. 18-38.

Rubio, L. 1957-1966: P. Terencio, Comedias, Barcelona, I-III.

Ruiz Arzalluz, I. 2007-2008 [2012]: «Petrarca, el texto de Terencio y Pietro da Moglio», Quaderni petrarcheschi 17-18, pp. 765-812.

Sabbadini, R. 1897: «Biografi e commentatori di Terenzio», Studi Italiani di Filologia Classica 5, pp. 289-327.

Schlee, F. 1893: Scholia Terentiana, Leipzig.

Schorsch, F. 2011: Das commentum Monacense zu den Komödien des Terenz. Eine Erstedition des Kommentars zu 'Andria', 'Heautontomorumenos' und 'Phormio', Tubinga.

Silagi, G. y Bischoff, B. 1979: Monumenta Germaniae Historica. Poetae Latini medii aeui. V. 3. Die Ottonenzeit. Ergänzungen, Nachträge, Register, Múnich.

Umpfenbach, F. 1870: P. Terenti Comoediae, Berlín.

Velaza, J. 2007: La historia del texto de Terencio en la Antigüedad, Barcelona.

Victor, B. 1996: «A Problem of Method in the History of Texts and its Implications for the Manuscript Tradition of Terence», Revue d'Histoire des Textes 26, pp. 269-287.

Victor, B. 2003: «Simultaneous Copying of Classical Texts 800-1100: Techniques and Their Consequences», en Spilling, H. (ed.), La collaboration dans la production de l'écrit medieval. Actes du XIII colloque du comité international de paléographie latine (Weingarten, 22-25 septembre 2000), París, pp. 347-358.

Victor, B. 2007: «New Manuscript Sources of the Terence-Text», en Kruschwitz, P. W., Ehlers, W. y Felgentreu, F. (eds.), Terentius poeta, Múnich, pp. 1-14.

Victor, B. y Quesnel, B. 1999: «The Colometric Evidence for the History of the Terence-text in the Early Middle Ages», Revue d'Histoire des Textes 29, pp. 141168.

Villa, C. 1979: «'Denique Terenti dultia legimus acta ...': una 'lectura Terenti' a S. Faustino di Brescia nel secolo IX», Italia Medioevale e Umanistica 22, pp. 1-44. 
Villa, C. 1984: La 'Lectura Terentii'. I. Da Ildemaro a Francesco Petrarca, Padua. Webb, R. H. 1911: «An Attempt to Restore the $\gamma$ Archetype of Terence Manuscripts», Harvard Studies in classical Philology 22, pp. 55-110.

SiGLAS $^{30}$

A: Città del Vaticano, Biblioteca Apostolica Vaticana, Vat. lat. 3226 (s. IV/V, probablemente Italia).

b: Bologna, Biblioteca Universitaria di Bologna, 4329 bis (s. XV, Italia).

C: Città del Vaticano, Biblioteca Apostolica Vaticana, Vat. lat. 3868 (s. IX ${ }^{2 / 4}$, Francia).

Conv: Firenze, Biblioteca Medicea Laurenziana, Conv. soppr. 510 (s. XII y XIV, Italia).

D: Firenze, Biblioteca Medicea Laurenziana, 3824 (s. X/XI [ff. 5-6, 13-14, 106, 128: s. XI], Alemania o Francia).

E: Firenze, Biblioteca Riccardiana, 528 (s. X/XI [ff. 1-2: s. XIV], tal vez Alemania).

e: El Escorial, Biblioteca del Real Monasterio, S III 23 (s. X/XI in. [ff. 9-16: s. XII], Italia $\left.{ }^{31}\right)$.

F: Milano, Biblioteca Ambrosiana, H 75 inf. (s. IX/X [ff. 21-22, 99-100: s. XI], Francia).

Fi: Firenze, Biblioteca Medicea Laurenziana, S. Marco 244 (s. XII, Italia)

G: Città del Vaticano, Biblioteca Apostolica Vaticana, Vat. lat. 1640 (s. X, Alemania).

L: Leipzig, Universitätsbibliothek, Rep. I 37 (s. X, tal vez Alemania).

M: München, Bayerische Staatsbibliothek, Clm 14420, ff. 79-144 (s. XI in., presumiblemente Brescia).

Ma: Milano, Biblioteca Ambrosiana, G 130 inf. (s. XI y XIV, sur de Italia).

Mp: Montpellier, Bibliothèque Interuniversitaire de Montpellier, Section de médécine, H 332 (a. 1370, Italia).

Ot: Città del Vaticano, Biblioteca Apostolica Vaticana, Ottob. lat. 1031 (s. XI, Alemania).

${ }^{30}$ El siguiente elenco incluye los mss. que se mencionan en el presente artículo. La lista está dispuesta alfabéticamente según las siglas, que corresponden, en la medida de lo posible, a las utilizadas en la bibliografía precedente: en concreto, Umpfenbach 1870, Kauer, Lindsay y Skutsch 1988, Marouzeau 1941-1949, Prete 1954, Rubio 1957-1966, Grant 1986, Velaza 2007, Billanovich 1974 y Ruiz Arzalluz 2007-2008. Junto a la signatura del ms. se señalan solo los datos estrictamente indispensables, para los cuales nos hemos atenido a la bibliografía existente sobre cada uno de ellos.

${ }^{31}$ Riou 1978, p. 8. Munk Olsen 1982-2009, II, p. 607, sitúa su origen en España. 
P: Paris, Bibliothèque nationale de France, lat. 7899 (s. IX [ff. 29-30: s. IX/X], Francia).

p: Paris, Bibliothèque nationale de France, lat. 10304 (s. X/XI, Francia).

Pc: Paris, Bibliothèque nationale de France, lat. 7900A (s. IX/X, Milán).

Pm: Parma, Biblioteca Palatina, Palat. 1661 (a. 1470, Italia).

v: Valenciennes, Bibliothèque Municipale, 448 (420) (s. X/XI, Francia).

Vb: Wien, Österreichische Nationalbibliothek, 85-II (s. X/XI in., sur de Alemania). Wog: Wolfenbüttel, Herzog-August-Bibliothek, Cod. Guelf. 22 Gud. Lat. (s. XV ex., Alemania).

Y: Paris, Bibliothèque nationale de France, lat. 7900 (s. IX med., Corbie).

$\varepsilon$ : Einsiedeln, Stiftsbibliothek, 362-II (s. X, Alemania o Suiza).

$\eta$ : Einsiedeln, Stiftsbibliothek, 362-I (s. X, Alemania, Suiza o Francia). 